\title{
Guida in stato di alterazione psico-fisica: aspetti medico-legali relativi ai cannabinoidi
}

\author{
Elio Santangelo ${ }^{1}$
}

1 Medico-Chirurgo, Specialista in Medicina Legale e delle Assicurazioni. Dirigente Medico, Struttura Complessa di Medicina Legale, Azienda Ospedaliero-Universitaria, Ospedali Riuniti di Trieste

Driving while impaired: medico-legal aspects related to cannabinoids

Pratica Medica \& Aspetti Legali 2014; 8(2): 39-42

http://dx.doi.org/10.7175/PMeAL.v8i2.919

Come l'uso di Cannabis è aumentato negli ultimi anni, così è cresciuta la necessità di migliorare le metodologie e le strategie mediante le quali sia possibile studiarne l'impatto sulla salute.

Oggi l'analisi dei cannabinoidi nei liquidi biologici è oggetto di notevole interesse pubblico e rappresenta anche una enorme sfida scientifica che abbisogna, da un lato, di poter disporre di indagini tossicologiche sempre più sensibili e affidabili per affrontare questo studio, dall'altro di incrementare le conoscenze circa la sostanza e gli effetti che è in grado di determinare, al fine di poter interpretare adeguatamente gli esiti delle analisi.

In merito alla guida di veicoli, una delle problematiche di maggiore interesse riguarda la relazione che intercorre tra i valori di concentrazione di $\Delta^{9}$-THC misurati su campione ematico e il presunto stato di intossicazione del soggetto cui il campione appartiene. Nel tentativo di determinare se sia possibile stabilire un livello soglia di $\Delta^{9}$-THC oltre il quale si venga a realizzare una condizione di disabilità, sono stati avviati da parte dello statunitense National Istitute of Drug Abuse (NIDA) studi finalizzati alla valutazione degli effetti indotti da differenti dosi di Cannabis, somministrate a volontari, sui compiti e sulle abilità correlati alla guida. I risultati forniti da tale indagine suggeriscono che, mentre appare possibile stimare la relazione esistente tra dosi assunte e concentrazioni attese, la valutazione tra dosi e performance risulta caratterizzata da maggiore incertezze [1].

In aggiunta, anche se si può far conto su di un campione di liquido biologico prelevato al soggetto, né l'urina, né la saliva, né il sangue possono produrre una misurazione senza riserve della presenza e quantificazione del grado di disabilità alla guida, specifico per il soggetto indagato.

Uno dei motivi alla base di tale incertezza è rappresentato dalla complessità delle caratteristiche farmacodinamiche e farmacocinetiche della sostanza.

Una interpretazione approfondita circa la relazione esistente tra concentrazione riscontrata nei fluidi biologici e sussistenza e grado degli effetti determinati sul comportamento necessiterebbe di integrare tra loro informazioni riguardanti diversi aspetti relativi alla sostanza assunta, quali il profilo farmacologico, variabile da individuo a individuo in funzione di caratteristiche genetiche, fisiologiche e ambientali [2]. Di rado tutte queste informazioni risultano complessivamente disponibili in casi di pertinenza tossicologico-forense e non solo.

Anche se è ben compreso che l'assunzione di droghe può risultare dannosa per una guida sicura, la misura in cui farmaci e/o sostanze stupefacenti possono deteriorare le prestazioni di guida è spesso complicata da prevedere [3]. Di conseguenza, com-

Corresponding author Elio Santangelo dotteliosantangelo@gmail.com

\section{Disclosure}

II presente articolo è un estratto del volume "Guida in stato di alterazione psico-fisica: I'esempio della Cannabis", dello stesso autore 
prendere la relazione tra valori di concentrazione rilevati in un unico campione biologico e grado di disabilità prodotto rimane a tutt'oggi un compito non semplice [4]. Le molteplici variabili coinvolte possono infatti determinare un ampio spettro di possibili interpretazioni.

Prendendo in considerazione sostanze stupefacenti e sostanze psicotrope, formulare e sostenere una accusa, da un punto di vista scientifico $\mathrm{e}$, più specificamente medico-legale, in merito ad una condizione di disabilità, appare più complesso rispetto a quanto accade nel caso della guida sotto l'influenza dell'alcool. Natura e grado della disabilità possono essere difficili da distinguere e provare, rendendo di conseguenza meno agevole la persecuzione di tali casi.

Anche se l'alcool è una droga, non tutte le droghe possono essere considerate allo stesso modo dell'alcool. Ciò significa che nel caso in cui un conducente sia sospettato di guida sotto l'effetto di sostanze stupefacenti, anche definita DUID (Driving Under Influence of Drugs) [5], è possibile che siano richiesti un trattamento e una valutazione speciali per definire la sussistenza del reato. Una buona interazione e una effettiva integrazione tra forze dell'ordine ed esperti dell'ambito legale e scientifico risulta fondamentale in questi casi. Dato che la guida di un autoveicolo rappresenta un'attività complessa che, per essere svolta, comporta il possedere abilità psico-fisiche ben determinate, si considera che l'assunzione di qualsiasi farmaco o sostanza capace di influenzare i fisiologici processi mentali e/o fisici sia in grado di ridurre le performance necessarie alla guida. Il livello di compromissione della funzionalità e della performance del soggetto dipende dalle caratteristiche della sostanza, dalle modalità di assunzione, dal tempo intercorso tra utilizzo della sostanza e campionamento della matrice biologica per l'effettuazione delle relative analisi.

Queste fonti di variabilità, associate ai costi legati ai test tossicologici, alle differenti risorse disponibili da Stato a Stato e ai diversi protocolli utilizzati dai laboratori rendono il problema ancora più complesso.

Dimostrare e definire una condizione di disabilità droga-correlata richiede oggi di sviluppare e affinare la comprensione degli effetti prodotti da sostanze specifiche e la loro potenzialità nel determinare effetti negativi sulla condotta di guida. La valutazione in ambito medico e statistico riguardante la prevalenza di droghe varia non soltanto in rapporto ad aree geografiche ma anche in relazione alle metodologie impiegate, al loro grado di sensibilità e specificità, e alla natura dei campioni utilizzati (sangue, urine, saliva, altro).

A fini interpretativi, inoltre, riveste un ruolo importante il periodo di tempo (detection time) in cui una sostanza o i suoi metaboliti possono essere presenti all'interno di un campione biologico. Esso varia notevolmente in funzione di: sostanza, matrice biologica esaminata e caratteristiche individuali del soggetto [6].

È importante considerare che, oltre ai risultati che possono emergere dalle analisi tossicologiche, ulteriori informazioni che includono le caratteristiche di guida, test riguardanti la performance, test psicofisici, comportamenti inusuali, condizioni del guidatore sono spesso necessarie, in ambito medico-legale, al fine del riconoscimento e della valutazione del nesso di causa tra consumo di sostanze psicoattive e sussistenza di disabilità a carico della persona indagata.

Al fine di fornire una valida testimonianza, in ambito tossicologico sarebbe sempre auspicabile poter associare ai risultati derivanti dalle analisi strumentali procedure cliniche unanimemente riconosciute e validate che forniscano elementi quanto più obiettivi in merito all'esame del soggetto, del comportamento tenuto e degli effetti clinicamente obiettivabili indotti dalla sostanza assunta. In tal senso, la messa a punto e il conseguente utilizzo di un approccio d'interpretazione multidisciplinare potrebbe essere di grande utilità, dato che la sola presenza di una sostanza psicoattiva in un campione biologico fornisce informazioni molto preziose ma, spesso, anche altri elementi devono essere tenuti in considerazione.

Fatta eccezione per l'etanolo, tra la comunità scientifica non esiste ancora una unanime posizione circa la possibilità di correlare determinate concentrazioni ematiche di una certa sostanza psicoattiva (come può essere il caso della Cannabis) agli effetti prodotti sulla guida e ai quadri di diminuzione di performance e disabilità corrispondenti alla definizione di "guida in stato di alterazione psico-fisica» [7].

I valori di concentrazione dei metaboliti, come l'11-OH-THC, correlano temporalmente con la presenza degli effetti psicoattivi ma non sono prontamente rintracciabili nel sangue, mentre i livelli ematici di $\Delta 9$-THC correlano solo in modo modesto con l'attualità dello stato di intossicazione, in virtù della natura liposolubile di tale molecola.

Secondo alcuni Autori tale condizione potrebbe, realisticamente, essere associata alla somma delle concentrazioni di tutti i cannabinoidi con attività psicoattiva presenti nei liquidi biologici, a livello cerebrale e negli altri tessuti.

In conclusione, per chi è chiamato a interpretare i dati analitici e anche per coloro cui competono valutazioni di carattere giuridico, appare importante poter disporre di approcci di studio volti ad indagare molteplici aspetti quali:

- Considerazioni empiriche: qual è la farmacologia della sostanza? Quali effetti produce? Per quanto tempo si prolunga l'effetto?

- Studi epidemiologici: studi retrospettivi che abbiano indagato sul rapporto tra sostanze/ comportamenti alla guida in una data popolazione di conducenti. 
- Valutazione di casi clinici: rapporti pubblicati in letteratura riguardanti definizione e valutazione dello stato di alterazione in relazione alla guida di autoveicoli, attraverso il riconoscimento obiettivato di quadri clinici ascrivibili ad assunzione di specifiche sostanze stupefacenti.

- Studi di laboratorio: somministrazione di una sostanza e valutazione in ambiente controllato del tempo di risposta agli stimoli visivi e uditivi, del controllo motorio, della memoria, della visione, degli effetti sull'umore, attraverso prove psico-fisiche.

- Studi al simulatore: somministrazione di una sostanza e valutazione delle performance mediante simulatore di guida. Possono essere indagate posizione mantenuta nella corsia, velocità e direzione assunte.

- Studi riguardanti la guida: somministrazione di una sostanza e valutazione delle prestazioni di guida in un ambiente e in condizioni "reali".

La guida in stato di alterazione psico-fisica per uso di sostanze stupefacenti e/o psicotrope continua a rimanere un problema attuale e socialmente sentito.

In Italia, la definizione ed evoluzione di normative finalizzate alla prevenzione e repressione del reato previsto dall' art. 187 del Codice della Strada ha visto negli anni l'introduzione di nuovi interventi legislativi relativamente alle metodiche di accertamento e agli aspetti sanzionatori.
Da una prospettiva strettamente medico-legale, la criteriologia presa in considerazione in casi di intossicazione dovrebbe prevedere la valutazione di parametri di natura clinica (relativamente alla sintomatologia presentata), anamnestica (valutazione dei risultati ottenuti in riferimento delle cause della fenomenologia considerata) e chimico-tossicologica (dimostrazione analitica della presenza nei liquidi biologici di un determinato tossico e dei suoi metaboliti) [8].

La risultanza di tutti gli elementi sopra considerati non sempre e non facilmente è disponibile. Come previsto dai chiarimenti del Ministero dell'Interno circa la procedura di accertamento del reato (richiamati in precedenza), l'introduzione di accertamenti clinici da affiancare a determinazioni di natura chimico-tossicologica non sarà di semplice esecuzione per il personale che verrà indicato all'assoluzione di tale ufficio, che presumibilmente dovrebbe avere caratteristiche proprie di un'attività di tipo tossicologico-clinico [9].

Nonostante, quindi, le molte pubblicazioni scientifiche ad oggi disponibili relative allo studio degli effetti che alcune classi di farmaci e le sostanze stupefacenti sono capaci di determinare sulle capacità di guida, da un punto di vista normativo e procedurale non è stato ancora raggiunto un approccio univoco che fornisca, alle parti chiamate in causa in contesti legati alla guida in stato di alterazione, strumenti realmente efficaci nel consentire il riconoscimento "certo" della sussistenza delle condizioni di disabilità correlate all'assunzione di sostanze ad azione stupefacente e/o psicotropa.

\section{BIBLIOGRAFIA}

1. National Institute on Drug Abuse (NIDA). The analysis of cannabinoids in biological fluids. Research monograph 1982; 42 : 4

2. Nassar AF, Hollenberg PF, Scatina J. Drug metabolism handbook. Concepts and applications. Hoboken: Wiley, 2009

3. Kerrigan S. Drug toxicology for prosecutors. Targeting hardcore impaired drivers. American Prosecutors Research Institute (APRI), 2004

4. Walsh JM. Illegal drugs and driving. International Council on Alcohol, Drugs and Traffic Safety (ICADTS). Working Group Report, 2000

5. Haley J. The truth about drugs. New York: DWJ BOOKS LLC, 2009

6. Sloboda Z. Epidemiology of drug abuse. New York: Springer-Vergas, 2005

7. Gotz W, Sznitman S, Olsson B. A Cannabis reader: global issues and experience. European Monitoring Centre for Drugs and Drug Addiction (EMCDDA), 2008

8. Baima Bollone P. Medicina legale. Torino: Giappichelli Editore, 2005

9. De Ferrari F, Palmieri L. Manuale di medicina legale, per una formazione, per una conoscenza. Milano: Giuffrè Editore, 2007 
Per chi desidera approfondire

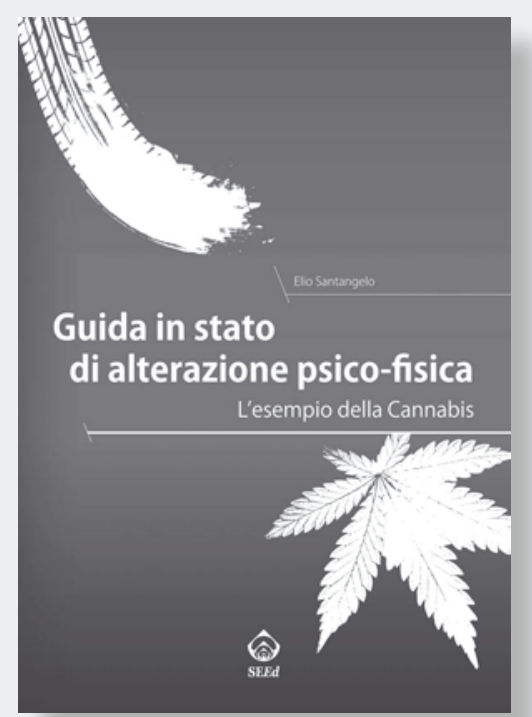

Guida in stato di alterazione psico-fisica: l'esempio della Cannabis

Autore: Elio Santangelo

Prezzo:

- $12,00 €$ (cartaceo)

- $8,49 €$ (ebook)

ISBN:

- 978-88-9741-953-2 (cartaceo)

- 978-88-9741-954-9 (ebook)

Acquistabile su www.edizioniseed.it

Prima edizione maggio 2014

La Cannabis rappresenta la sostanza illegale più frequentemente utilizzata nel mondo ed è quella maggiormente abusata da parte di guidatori che riferiscono di aver guidato dopo aver fatto uso di sostanze stupefacenti. Dal momento che sia la Cannabis che l'alcool possono influenzare le funzioni cognitive, spesso è assunto che l'uso di Cannabis condizioni negativamente la performance di guida parimenti a quanto avviene per l'alcool: mentre il grado di disabilità correlato all'assunzione di alcool può essere stimato sulla base della concentrazione ematica misurata,

non è stata invece del tutto accertata una chiara relazione tra le concentrazioni di cannabinoidi rilevate nel sangue o nella saliva e il corrispondente grado di disabilità.

II volume si propone di delineare lo stato dell'arte sulla normativa italiana in tema di guida in stato di alterazione per uso di stupefacenti, approfondendo le indicazioni per la ricerca tossicologica su materiale biologico e la relativa interpretazione medico-legale del dato tossicologico. 